\title{
Radial Pulsation of the roAp Star HD 122970?
}

\author{
G. Handler ${ }^{1,2}$, E. Paunzen ${ }^{1}$, R. Garrido ${ }^{3}$, J. A. Guzik ${ }^{4}$, T. E. Beach ${ }^{5}$, \\ R. Medupe ${ }^{6}$, F. Chagnon ${ }^{7}$, R. R. Shobbrook ${ }^{8}$, J. M. Matthews ${ }^{7}$, \\ T. A. Ryabchikova ${ }^{1,9}$, A. P. Hatzes ${ }^{10}$
}

\begin{abstract}
We acquired multisite observations of the newly discovered rapidly oscillating Ap star HD 122970. A frequency analysis shows the presence of two independent modes, one singlet and one equally spaced triplet. By applying the oblique pulsator model and asymptotic theory we infer that the triplet originates from a distorted dipole mode and that the singlet most probably corresponds to a radial mode.
\end{abstract}

\section{Introduction}

HD 122970 is an equatorial $8^{\text {th }}$ magnitude F0 star whose Strömgren indices indicate enhanced metallicity and line blocking. It was tested for photometric variability during a Northern Hemisphere survey for rapidly oscillating Ap stars (Handler \& Paunzen 1999) and it was discovered to be variable with a period of about $11 \mathrm{~min}$. Since this result was obtained at the beginning of a large multisite campaign on the $\delta$ Scuti star XX Pyx (see Arentoft et al. 2000), it was decided to include HD 122970 as a second-priority target in that campaign.

\section{Observations and Frequency Analysis}

We acquired $119 \mathrm{hr}$ of high-speed time-series photometry with seven telescopes at six observatories. The total time base of the measurements is five months, but $92 \%$ of the data was gathered during a six-week period.

The frequency analysis of the reduced $B$-filter light curves yielded four statistically significant periodicities (Table 1 ). Three of them $\left(f_{2}-f_{4}\right)$ form an equally spaced triplet within the accuracy of frequency determination.

\footnotetext{
${ }^{1}$ Institut für Astronomie, Universität Wien, Türkenschanzstraße 17, A-1180 Wien, Austria

${ }^{2}$ South African Astronomical Observatory, P.O. Box 9, Observatory 7935, South Africa

${ }^{3}$ Instituto de Astrofisica de Andalucia, Apt. 3044, E-18080 Granada, Spain

${ }^{4}$ Los Alamos National Laboratory, XTA, MS B220, Los Alamos, NM 87545-2345, USA

${ }^{5}$ University of New Mexico, Los Alamos, 4000 University Drive, Los Alamos, NM 87544, USA

${ }^{6}$ Department of Astronomy, University of Cape Town, Rondebosch 7700, South Africa

${ }^{7}$ Department of Physics and Astronomy, University of British Columbia, Vancouver, B.C. V6T 1Z4, Canada

${ }^{8}$ P. O. Box 518, Coonabarabran, N.S.W. 2357, Australia

${ }^{9}$ Institute of Astronomy, Pyatnitskaya Street 48, Moscow 109017, Russia

${ }^{10} \mathrm{Dept}$. of Astronomy and McDonald Observatory, University of Texas, Austin, TX 78712, USA
} 
Table 1. Multifrequency solution for the roAp star HD 122970. Phases are relative to pulsation amplitude maximum.

\begin{tabular}{ccccc}
\hline & $\begin{array}{c}\text { Frequency } \\
(\mu \mathrm{Hz})\end{array}$ & $\begin{array}{c}\text { B Amplitude } \\
(\mathrm{mmag})\end{array}$ & $\begin{array}{c}\text { Phase } \\
\text { (radians) }\end{array}$ & S/N \\
\hline$f_{1}$ & $1502.476 \pm 0.001$ & $1.68 \pm 0.03$ & $3.43 \pm 0.05$ & 29.8 \\
$f_{2}$ & $1477.823 \pm 0.002$ & $0.85 \pm 0.03$ & $1.87 \pm 0.10$ & 14.4 \\
$f_{3}$ & $1476.799 \pm 0.006$ & $0.43 \pm 0.03$ & $1.26 \pm 0.21$ & 7.3 \\
$f_{4}$ & $1478.929 \pm 0.010$ & $0.25 \pm 0.03$ & $1.07 \pm 0.36$ & 4.2 \\
\hline
\end{tabular}

\section{Interpretation}

By applying the oblique pulsator model (Kurtz 1982) and simple asymptotic theory to the observed pulsations, we can immediately rule out nine of the twelve initially possible mode identifications (assuming $\ell \leq 2$ ) for the two independent modes.

When imposing the measured $v \sin i$ and the rotation period from the triplet spacing as further constraints, one can again use the oblique pulsator model to infer limits on the "permitted" inclination angle of the rotational axis to the line of sight. This eliminates one further identification. Among the remaining two $\ell$ pairs the triplet is always a (distorted) $\ell=1$ mode and the singlet $f_{1}$ is either $\ell=0$ or $\ell=2$. However, the interpretation involving an $\ell=2$ identification for $f_{1}$ is only possible within a very small range of $i$, hence unlikely.

More observations of HD 122970 are needed to obtain a unique mode identification (an upper limit on possible rotational sidelobe amplitudes of $f_{1}$ lower than $0.1 \mathrm{mmag}$ is required), and to determine the degree of distortion of the conjectured radial mode compared to that of the dipole mode. This could help to understand the influence of the magnetic field on the pulsations.

See Handler et al. (2000) for further details.

\section{References}

Arentoft, T., Handler, G., Shobbrook, R. R., et al. 2000, in these proceedings, p. 469

Handler, G. \& Paunzen, E. 1999, A\&AS, 135, 57

Handler, G. et al. 2000, MNRAS, in preparation

Kurtz, D. W. 1982, MNRAS, 200, 807 